ИЗВЕСТИЯ АКАДЕМИИ НАУК ЭСТОНСКОН ССР. ТОМ Х СЕРИЯ ФИЗИКО-МАТЕМАТИЧЕСКИХ И ТЕХНИЧЕСКИХ НАУК. 1961, № 3

\title{
О ДИНАМИЧЕСКИХ ОШИБКАХ ДВУХКООРДИНАТНЫХ СЛЕДЯЩИХ СИСТЕМ С ПРОГРАММНЫМ УПРАВЛЕНИЕМ*
}

\section{К. ЛЕПпиК}

Для воспроизведения на выходе двухкоординатной следящей системы (CС) с программным управлением кривой $F(x, y)$ надо, во-первых, синтезировать программирующее устройство (ПУ), которое дает функцию $F(x, y)$ в параметрической форме $x=f_{1}(t), y=f_{2}(t)$. Функции $f_{1}(t)$ и $f_{2}(t)$ и будут входными воздействиями CC.

Если $\Phi_{x}(s)$ и $\Phi_{y}(s)$ - передаточные функции по координатам $x$ и $y$, тогда изображения выходных координат

$$
\begin{aligned}
& x_{1}(s)=\Phi_{x}(s) \cdot x(s) \\
& y_{1}(s)=\Phi_{y}(s) \cdot y(s),
\end{aligned}
$$

где $x(s)$ и $y(s)$ - изображения входных координат.

Оригиналы можем писать так:

$$
\begin{aligned}
& x_{1}=f_{1}(t)+\Delta f_{1}(t)+\Delta f_{1}^{\prime}(t) \\
& y_{1}=f_{2}(t)+\Delta f_{2}(t)+\Delta f_{2}^{\prime}(t),
\end{aligned}
$$

где $\Delta f(t)$ и $\Delta f^{\prime}(t)$ - ошибки воспроизведения.

Если $t \rightarrow \infty$, то $\Delta f^{\prime}(t) \rightarrow 0$.

Исключив $t$, получим уравнение воспроизведенной кривой $F^{\prime}(x, y)$.

Динамическая ошибка двухкоординатной следящей системы

$$
\delta=\left|F(x, y)-F^{\prime}(x, y)\right| .
$$

В данной статье рассматриваются динамические ошибки при восприизведении а) прямых линий и кривых второго порядка, б) парабол третьей и четвертой степени. Предполагается, что СС линейные и непрерывные и ПУ тоже непрерывного действия.

Предполагаем, что желаем воспроизвести кривую второго порядка

$$
F(x, y)=A x^{2}+2 B x y+C y^{2}+2 D x+2 E y+F=0 .
$$

Соответствующее ПУ описывается дифференциальными уравнениями [1]

$$
\begin{aligned}
& \frac{d x}{d t}= \pm \omega(B x+C y+E) \\
& \frac{d y}{d t}=\mp \omega(A x+B y+D) .
\end{aligned}
$$

* Работа выполнена в Институте электромеханики АН СССР под руководством доктора технических наук А. А. Воронова. 
Чтобы найти входные функции для СС $x=f_{1}(t)$ и $y=f_{2}(t)$, надо решить эту систему уравнений.

Характеристическое уравнение этой системы:

$$
s^{2}-\omega^{2}\left(B^{2}-A C\right)=0,
$$

где $B^{2}-A C-$ инвариант кривой второго порядка.

Если $B=0$, то оси кривых второго порядка становятся параллельными одной из координатных осей. Если $A=B=C=0$, то получим на выходе ПУ прямые. Такое ПУ, где $B=0$, описано в работе [ $\left.{ }^{1}\right]$ и изображено здесь на фиг. 1a. В дальнейшем предполагаем, что управление следящими системами производится от такого ПУ.

\section{Воспроизведение прямых линий}

На выходе ПУ имеем

$$
\begin{aligned}
& x=x_{0}+\omega y_{\mathrm{c}} t \\
& y=y_{0}+\omega x_{\mathrm{c}} t .
\end{aligned}
$$

Пусть СС описываются уравнениями

$$
\begin{aligned}
& \left(T_{2 x}^{2} s^{2}+T_{1 x} s+1\right) x_{1}=x \\
& \left(T_{2 y}^{2} s^{2}+T_{1 y} s+1\right) y_{1}=y .
\end{aligned}
$$

Если у нас, например, передаточные функции СС (фиг. 1б) $W(s)=$ $=\frac{k}{(T s+1) s}$, то постоянные в уравнениях (2) будут

$$
T_{2}^{2}=\frac{T}{k}, \quad T_{1}=\frac{1}{k} .
$$

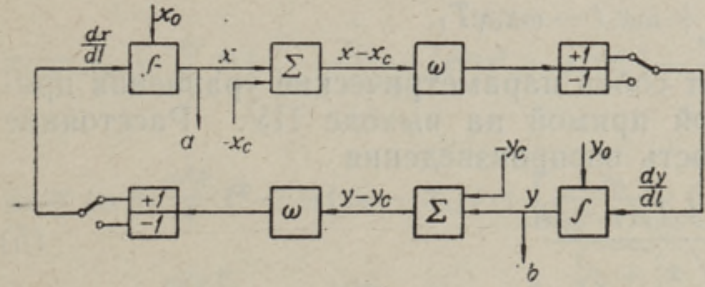

$a$

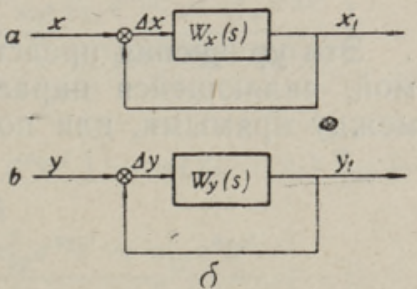

$\delta$

Фнг. 1. $a$ - программирующее устройство (ПУ) для воспроизведения кривых второго порядка и прямых; б - следящие системы (СС).

Решая уравнения (2) с входными воздействиями (1), получим $x_{1}=x_{0}+\omega y_{\mathrm{c}} t-\omega y_{\mathrm{c}} T_{1 x}+\frac{\dot{x_{0}}}{a_{1 x}-a_{2 x}}\left(e^{\alpha_{1} x^{t}}-e^{\alpha_{2 x} t}\right)+\omega y_{\mathrm{c}} \frac{a_{2 x}^{2} e^{\alpha_{1} x^{t}}-a_{1 x}^{2} e^{\alpha_{2 x} t}}{\left(a_{1 x}-a_{2 x}\right) a_{1 x} a_{2 x}}$ $y_{1}=y_{0}+\omega x_{\mathrm{c}} t-\omega x_{\mathrm{c}} T_{1 y}+\frac{\dot{y}_{0}}{a_{1 y}-\alpha_{2 y}}\left(e^{\alpha_{1} t}-e^{\alpha_{2 y} t}\right)+\omega x_{\mathrm{c}} \frac{a_{2 y}^{2} e^{\alpha_{1} y^{t}}-a_{1 y}^{2} e^{\alpha_{2} t}}{\left(a_{1 y}-a_{2 y}\right) a_{1 y} \alpha_{2 y}}$

Здесь $a-$ корни характеристических уравнений в каналах $x$ и $y$; $x_{0}, y_{0}, \dot{x}_{0}, \dot{y}_{0}$ - начальные условия. 
Если следящие системы идентичные, то $\alpha_{1 x}=\alpha_{1 y}=\alpha_{1}, \alpha_{2 x}=\alpha_{2 y}=\alpha_{2}$ и $T_{1 x}=T_{1 y}=T_{1}, T_{2 x}^{2}=T_{2 y}^{2}=T_{2}^{2}$.

Очевидно, что прямая воспроизводится без погрешности, если следящие системы идентичны и $\dot{x}_{0}=\dot{y}_{0}=0$ или $\dot{x}_{0}=\frac{y_{\mathrm{c}}}{x_{\mathrm{c}}} \dot{y}_{0}$. При этом $x \neq x_{1}$ и $y \neq y_{1}$, но $\frac{\Delta x}{\Delta y}=\frac{y_{\mathrm{c}}}{x_{\mathrm{c}}}$.

Если $\dot{x}_{0}$ и $\dot{y}_{0}-$ произвольные величины, тогда получим следующую формулу для погрешности:

$$
\delta=\frac{\dot{x}_{0} x_{\mathrm{c}}-\dot{y}_{0} y_{\mathrm{c}}}{\sqrt{x_{\mathrm{c}}^{2}+y_{\mathrm{c}}^{2}}} \cdot \frac{e^{a_{1} t}-e^{\alpha_{2} t}}{a_{1}-a_{2}} .
$$

Следовательно, для того, чтобы найти максимальную ошибку при заданных $\dot{x}_{0}$ и $\dot{y}_{0}$, надо найти максимум функции

$$
f(t)=\frac{e^{\alpha_{1} t}-e^{a_{2} t}}{a_{1}-a_{2}} .
$$

В [ $\left.{ }^{1}\right]$ доказано, что $f(t)_{\max } \approx \frac{1,05 T_{2}^{2}}{T_{1}+T_{2}}$.

Рассмотрим влияние неидентичности СС, пренебрегая переходным процессом. Возьмем $T_{1 x}=T_{1}, T_{2 x}^{2}=T_{2}^{2}, T_{1 y}=\gamma T_{1}, T_{2 y}^{2}=\gamma T_{2}^{2}$. Получим

$$
\begin{gathered}
x_{1}=x_{0}+\omega y_{\mathrm{c}} t-\omega y_{\mathrm{c}} T_{1} \\
y_{1}=y_{0}+\omega x_{\mathrm{c}} t-\omega x_{\mathrm{c}} \gamma T_{1} .
\end{gathered}
$$

Эти уравнения представляют собой параметрические уравнения прямой, являющейся параллельной прямой на выходе ПУ. Расстояние между прямыми, или погрешность воспроизведения

$$
\delta=\frac{\omega x_{\mathrm{c}} y_{\mathrm{c}} T_{1}(\gamma-1)}{\sqrt{x_{\mathrm{c}}^{2}+y_{\mathrm{c}}^{2}}}
$$

или, учитывая, что $\omega=\frac{v}{\sqrt{x_{0}^{2}+y_{c}^{2}}}(v-$ скорость движения изображающей точки по прямой),

$$
\delta=\frac{v x_{\mathrm{c}} y_{\mathrm{c}} T_{1}(\gamma-1)}{x_{\mathrm{c}}^{2}+y_{\mathrm{c}}^{2}} .
$$

Очевидно, что эта погрешность максимальна при $x_{\mathrm{c}}=y_{\mathrm{c}}$, т. е. при наклоне $45^{\circ}$.

Если введем в ПУ вместо начальных условий $x_{0}$ и $y_{0}$ начальные условия

$$
\begin{aligned}
& X_{0}=x_{0}+\omega y_{\mathrm{c}} T_{1} \\
& Y_{0}=y_{0}+\omega x_{\mathrm{c}} T_{1},
\end{aligned}
$$

то на выходе СС получим 


$$
\begin{aligned}
& x_{1}=x_{0}+\omega y_{\mathrm{c}} t+\frac{\dot{x}_{0}-\omega y_{\mathrm{c}}}{\alpha_{1}-\alpha_{2}}\left(e^{\alpha_{1} t}-e^{\alpha_{2} t}\right) \\
& y_{1}=y_{0}+\omega x_{\mathrm{c}} t+\frac{\dot{y}_{0}-\omega x_{\mathrm{c}}}{\alpha_{1}-\alpha_{2}}\left(e^{\alpha_{1} t}-e^{\alpha_{2} t}\right) .
\end{aligned}
$$

Как видим, после окончания переходного процесса $x=x_{1}$ и $y=y_{1}$. Но ошибка в переходном процессе выражается тем же уравнением (4). Следовательно, введение корректирующих импульсов в программу при воспроизведении прямых не имеет практического значения в случае идентичных СС. Введение при неидентичных СС соответствующих начальных условий $X_{0}=x_{0}+\omega y_{\mathrm{c}} T_{1 x}$ и $Y_{0}=y_{0}+\omega x_{\mathrm{c}} T_{1 y}$ компенсирует ошибки при $t \rightarrow \infty$.

\section{Воспроизведение парабол}

Рассмотрим случай, когда ось параболы параллельна оси $y$.

Уравнение параболы $p\left(y-y_{\mathrm{c}}\right)=\left(x-x_{\mathrm{c}}\right)^{2} ; y_{\mathrm{c}}$ и $x_{\mathrm{c}}-$ координаты вершины параболы.

На выходе ПУ имеем

$$
\begin{aligned}
& x=x_{0}+\frac{\omega p}{2} t \\
& y=y_{0}+\omega\left(x_{0}-x_{\mathrm{c}}\right) t+\frac{\omega^{2} p}{4} t^{2} .
\end{aligned}
$$

Пусть следящие системы опять описываются уравнениями (2). Тогда при входных воздействиях (9) и начальных условиях $x_{0}, y_{0}, \dot{x}_{0}$ и $\dot{y}_{0}$ на выходе СС получим

$$
\begin{gathered}
x_{1}=x_{0}+\frac{\omega p}{2} t+\left[\frac{\dot{x}_{0}}{a_{1 x}-a_{2 x}}\left(e^{\alpha_{1} x^{t}}-e^{\alpha_{2} x^{t}}\right)-\frac{\omega p T_{1 x}}{2}+\frac{\omega p}{2} \frac{a_{2 x}^{2} e^{\alpha_{1} x^{t}}-a_{1 x}^{2} e^{\alpha_{2 x} t}}{\left(a_{1 x}-a_{2 x}\right) a_{1 x} a_{2 x}}\right] \\
y_{1}=y_{0}+\frac{\omega^{2} p}{4} t^{2}+\omega\left(x_{0}-x_{c}\right) t+\left[\frac{\dot{y}_{0}}{a_{1 y}-a_{2 y}}\left(e^{\alpha_{1} y^{t}}-e^{\alpha_{2} y^{t}}\right)-\omega\left(x_{0}-x_{\mathrm{c}}\right) T_{1 y}-\right. \\
-\frac{\omega^{2} p T_{1 y}}{2} t+\frac{\omega^{2} p}{2}\left(T_{1 y}^{2}-T_{2 y}^{2}\right)+\omega\left(x_{0}-x_{\mathrm{c}}\right) \frac{a_{2 y}^{2} e^{\alpha_{1} t}-a_{1 y}^{2} e^{\alpha_{2 y} t}}{\left(a_{1 y}-a_{2 y}\right) a_{1 y} c_{2 y}}+ \\
\left.+\frac{\omega^{2} p}{2} \frac{a_{2 y}^{3} e^{\alpha_{1} t}-a_{1 y}^{3} e^{\alpha_{2 y} t}}{\left(a_{1 y}-a_{2 y}\right) a_{1 y}^{2} \alpha_{2 y}^{2}}\right]
\end{gathered}
$$

Выражения в квадратных скобках - динамические ошибки по координатам $x$ и $y$.

После окончания переходного процесса получим

$$
\begin{aligned}
x_{1}=x_{0}+\frac{\omega p}{2} t+\left[-\frac{\omega p T_{1 x}}{2}\right] & \\
y_{1}=y_{0}+\omega\left(x_{0}-x_{\mathrm{c}}\right) t & +\frac{\omega^{2} p}{4} t^{2}+\left[\frac{\omega^{2} p}{2}\left(T_{1 y}^{2}-T_{2 y}^{2}\right)-\omega\left(x_{0}-x_{\mathrm{c}}\right) T_{1 y}-\right. \\
& \left.-\frac{\omega^{2} p T_{1 y}}{2} t\right] .
\end{aligned}
$$


Как видно, динамическая ошибка по координате $x$ является постоянной величиной, а динамическая ошибка по координате $y$ зависит от времени.

Возьмем $x_{\mathrm{c}}=y_{\mathrm{c}}=0$ (т. е. вершина параболы находится в начале координат) и предположим, что СС идентичные.

Исключая из (11) $t$, получим уравнение кривой на выходе СС. Тогда имеем

$$
y=\frac{x^{2}}{p}+\frac{\omega^{2} p}{4}\left(T_{1}^{2}-2 T_{2}^{2}\right)
$$

Это уравнение параболы, ось которой совпадает с осью параболы на выходе ПУ, а вершина находится в точке $x=0, y=\frac{\omega^{2} p}{4}\left(T_{1}^{2}-2 T_{2}^{2}\right)$ (фиг. 2).

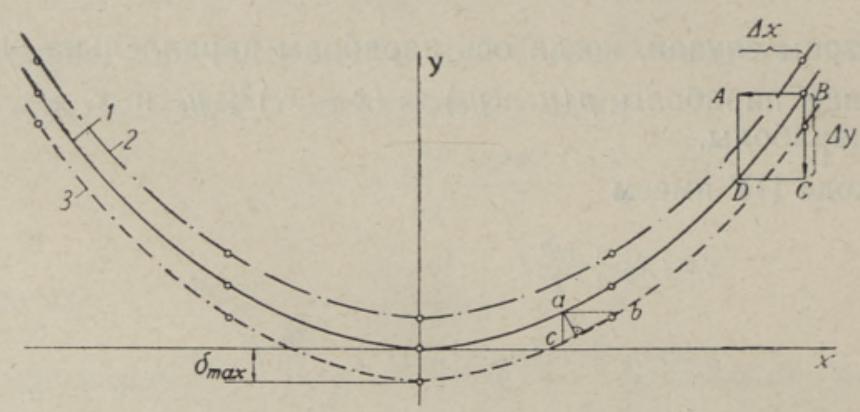

Фиг. 2. Параболы на выходе ПУ и СС при идентичных СС: 1 - на выходе ПУ; 2 - на выходе СС при $T_{1}^{2}>2 T_{2}^{2}$; 3 - на выходе СС при $T_{1}^{2}<2 T_{2}^{2}$.

Ошибка воспроизведения - это расстояние между параболами. Максимальным расстоянием будет расстояние между вершинами парабол. Это и является максимальной ошибкой.

$$
\delta_{\max }=\frac{\omega^{2} p}{4}\left(T_{1}^{2}-2 T_{2}^{2}\right) .
$$

Здесь $\omega=\frac{v}{\sqrt{y^{2}+\frac{p^{2}}{4}}}$.

Чтобы определить погрешность воспроизведения в любой точке параболы, надо найти выражение для расстояния между параболами. Так как точное выражение очень громоздкое, то оно здесь не приводится. Приближеннсе выражение для погрешности найдем из треугольника $a b c$ на фиг. 2. Там отрезок $a c \approx \delta$

$$
\delta=\frac{\Delta y^{\prime} \Delta x^{\prime}}{\sqrt{\left(\Delta y^{\prime}\right)^{2}+\left(\Delta x^{\prime}\right)^{2}}} \text {. }
$$

Здесь $\Delta y^{\prime}=\delta_{\max }, \quad \Delta x^{\prime}=x-\sqrt{x^{2}-p \Delta y^{\prime}}$.

Для данной параболы и данной СC $\Delta y^{\prime}=$ const $=\delta_{\max }$, а $\Delta x^{\prime}$. - переменная величина. 
Из (13) видно, что энак $\delta$ может быть плюс или минус. Если $T_{1}^{2}=2 T_{2}^{2}$, тогда погрешности нет. Это означает, что динамические ошибки по координатам $x$ и $y$ такие, что изображающая точка все время движется по заданной параболе (прямоугольник $A B C D$ на фиг. 2).

Чтобы охарактеризовать изменение $\delta_{\max }$ в зависимости от $T_{1}$ и $T_{2}^{2}$, обозначаем $\frac{T_{2}^{2}}{T_{1}^{2}}=\beta ;$ тогда получим

$$
\delta_{\max }=\frac{\omega^{2} p}{4}\left(\frac{1}{\beta^{2}}-\frac{2}{\beta}\right) \frac{T_{2}^{4}}{T_{1}^{2}} .
$$

Зависимость $f(\beta)=\frac{1}{\beta^{2}}-\frac{2}{\beta}$ изображена на фиг. 3 . Из рисунка видно, что ошибка имеет максимум при $\beta=1$ и что $f(\beta)$ проходит ноль с очень большим наклоном.

Фиг. 3. Зависимость ошибки от постоянных времени СС.

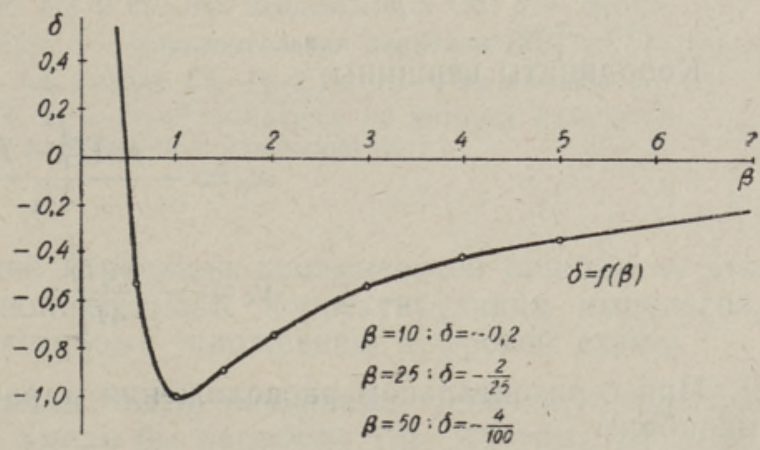

Для прнмера рассмотрим ошибки при замкнутой СС с астатизмом первого порядка. Пусть передаточная функция в разомкнутом состоянии $W=\frac{k}{(T s+1) s}$. Выбираем скорость движения по параболе $v=50$ мм $/$ сек; $p=20$ мм; $\omega=5$.

При разных $T$ и $k$ получим следующие погрешности (в мм):

\begin{tabular}{r|c|c|c|c}
\hline \multicolumn{1}{c|}{$T$} & 0,01 & 0,1 & 0,5 & 1 \\
\hline 100 & $-0,01$ & $-0,19$ & $-1,0$ & -2 \\
200 & $-0,0075$ & $-0,1$ & $-0,5$ & -1 \\
500 & $-0,0036$ & $-0,04$ & $-0,2$ & $-0,4$ \\
1000 & $-0,002$ & $-0,02$ & $-0,1$ & $-0,2$
\end{tabular}

Если следящие системы неидентичны $\left(T_{y}=\gamma T_{x}\right)$, то после окончания переходного процесса получим

$$
\begin{aligned}
& x_{1}=\frac{\omega p t}{2}-\frac{\omega p T_{1}}{2} \\
& y_{1}=\frac{\omega^{2} p t^{2}}{4}-\frac{\omega^{2} p \gamma T_{1} t}{2}-\frac{\omega^{2} p \gamma T_{2}^{2}}{2}+\frac{\omega^{2} p \gamma^{2} T_{1}^{2}}{2} .
\end{aligned}
$$


Исключая $t$, получим

$$
y=\frac{x^{2}}{p}+\omega T_{1}(1-\gamma) x+\frac{\omega^{2} p}{4}\left[T_{1}^{2}\left(1-2 \gamma+2 \gamma^{2}\right)-2 \gamma T_{2}^{21}\right] .
$$

Это парабола, ось которой параллельна оси $y$. Координаты вершины параболы

$$
\begin{aligned}
& x_{\mathrm{c}}=-\frac{\omega p T_{1}(1-\gamma)}{2} \\
& y_{\mathrm{c}}=\frac{\omega^{2} p}{4}\left(\gamma^{2} T_{1}^{2}-2 \gamma T_{2}^{2}\right) .
\end{aligned}
$$

Рассматриваем $\gamma$ как параметр и найдем уравнение кривой, на которой находится вершина параболы при изменении $\gamma$. Эта кривая является параболой с уравнением

$$
y=\frac{x^{2}}{p}+\omega x\left(T_{1}^{2}-T_{2}^{2}\right)+\frac{\omega^{2} p}{4}\left(T_{1}^{2}-2 T_{2}^{2}\right)
$$

Координаты вершины

$$
\begin{aligned}
& x_{\mathrm{c}}=-\frac{p \omega\left(T_{1}^{2}-T_{2}^{2}\right)}{2 T_{1}} \\
& y_{\mathrm{c}}=-\frac{\omega^{2} p T_{2}^{4}}{4 T_{1}^{2}}
\end{aligned}
$$

При горизонтальном расположении параболы при $T_{y}=\gamma T_{x}$ получим параболы

$$
x=\frac{y^{2}}{p}+\omega T_{1} y(\gamma-1)+\frac{\omega^{2} p}{4}\left[T_{1}^{2}\left(2+\gamma^{2}-2 \gamma\right)-2 T_{2}^{2}\right] .
$$

Координаты вершины параболы

$$
\begin{aligned}
& x_{\mathrm{c}}=\frac{\omega^{2} p}{4}\left(T_{1}^{2}-2 T_{2}^{2}\right) \\
& y_{\mathrm{c}}=-\frac{\omega p T_{1}(\gamma-1)}{2} .
\end{aligned}
$$

Как видно, при изменении $\gamma$ вершины находятся на прямой.

На фиг. 4 изображены параболы при различных $\gamma$. Из рисунка видно, что зависимость погрешности от $\gamma$ на различных участках параболы различна. Так, максимальная погрешность при вертикальной параболе при $\gamma<1$ растет (в сравнении с идентичными системами), а при $\gamma>1$ уменьшается. При горизонтальной параболе максимальная погрешность растет при $\gamma>1$ и при $\gamma<1$, но меньше, чем при $\gamma<1$ в случае вертикальной параболы. Самая большая ошибка при отработке левой ветви горизонтальной параболы при $\gamma<1$. При отработке правой ветви погрешности растут, если $\gamma>1$.

Здесь мы рассматривали только параболы, оси которых параллельны координатным осям. Но если бы мы имели ПУ, которое на выходе дает параболу с произвольным расположением осей, тогда можно бы- 
ло бы легко доказать, что при идентичных СС выражения ошибок такие же, как и при параболах, оси которых параллельны координатным осям. При неидентичных СС об изменении ошибки можно судить при помощи двух крайних случаев - вертикального и горизонтального расположения парабол.

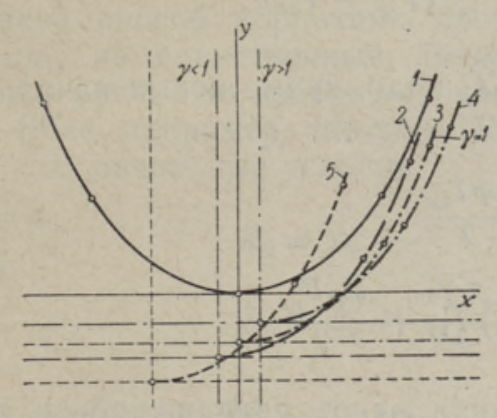

$\alpha$

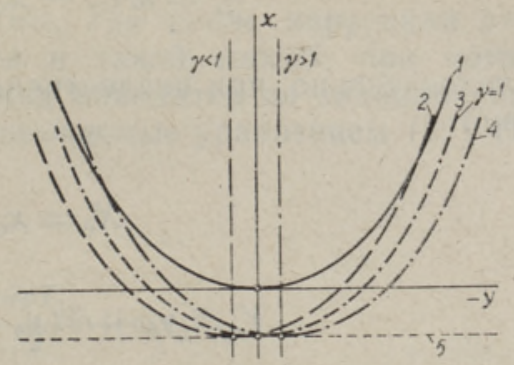

$\delta$

Фнг. 4. Параболы на выходе ПУ и СС при неидентинных СС: $a-$ вертикальная парабола $\left(2 T_{2}^{2}>T_{1}^{2}\right) ; \sigma-$ горизонтальная парабола $\left(2 T_{2}^{2}>T_{1}^{2}\right)$. 1 - на выходе Пу; 2 - на выходе СС $(\gamma<1) ; 3$ - на выходе СС $(\gamma=1) ; 4-$ на выходе СС $(\gamma>1) ; 5$ - кривая, на которой находятся вершины парабол при изменении $\gamma$.

Один из возможных путей коррекции динамической ошибки - это введение в программу дополнительных корректирующих импульсов, что, однако, приводит к некоторому усложнению цифровой схемы.

Посмотрим, какими должны быть начальные условия, вводимые в ПУ, чтобы на выходе СС имели бы параболу (9). Другими словами, надо найти такие начальные условия, при которых частное решение системы (2) было бы (9).

$$
\begin{aligned}
\left(T_{2 x}^{2} s^{2}+T_{1 x} s+1\right)\left(x_{0}+\frac{\omega p t}{2}\right) & =x \\
\left(T_{2 y}^{2} s^{2}+T_{1 y} s+1\right)\left(y_{0}+\omega\left(x_{0}-x_{c}\right) t+\frac{\omega^{2} p t^{2}}{4}\right) & =y .
\end{aligned}
$$

Отсюда получим требуемые входные воздействия

$$
\begin{aligned}
& x=x_{0}+\frac{\omega p t}{2}+\frac{\omega p T_{1 x}}{2} \\
& y=y_{0}+\omega\left(x_{0}-x_{\mathrm{c}}\right) T_{1 y}+\left[\frac{\omega^{2} p T_{1 y}}{2}+\omega\left(x_{0}-x_{\mathrm{c}}\right)\right] t+\frac{\omega^{2} p t^{2}}{4}+\frac{\omega^{2} T_{2 y p}^{2} p}{2} .
\end{aligned}
$$

Дифференцируем

$$
\begin{aligned}
& \frac{d x}{d t}=\frac{\omega p}{2} \\
& \frac{d y}{d t}=\omega\left(x_{0}-x_{\mathrm{c}}\right)+\frac{\omega^{2} p t}{2}+\frac{\omega^{2} T_{1 y} p}{2}
\end{aligned}
$$


Подставляя из (21) $t$, получим

$$
\begin{gathered}
\frac{d x}{d t}=\frac{\omega p}{2} \\
\frac{d y}{d t}=\omega\left(x-x_{\mathrm{c}}\right)+\frac{\omega^{2} p}{2}\left(T_{1 y}-T_{1 x}\right) .
\end{gathered}
$$

Следовательно, при неидентичных СС надо, кроме новых начальных условий

$$
\begin{gathered}
X_{0}=x_{0}+\frac{\omega p T_{1 x}}{2} \\
Y_{0}=y_{0}+\omega\left(x_{0}-x_{\mathrm{c}}\right) T_{1 y}+\frac{\omega^{2} p T_{2 y}^{2}}{2}
\end{gathered}
$$

(получим, полагая в $(21) t=0)$, ввести в ПУ еще $\frac{\omega^{2} p}{2}\left(T_{1 y}-T_{1 x}\right)$.

Но полное решение системы (2) при входных воздействиях

$$
\begin{gathered}
x=x_{0}+\frac{\omega p t}{2}+C_{1} e^{\alpha_{1} 1 x^{t}}+C_{2} e^{\alpha_{2} x^{t}} \\
y=y_{0}+\omega\left(x_{0}-x_{\mathrm{c}}\right) t+\frac{\omega^{2} p t^{2}}{4}+C_{3} e^{\alpha_{1} y^{t}}+C_{4} e^{\alpha_{2} y^{t}} .
\end{gathered}
$$

Коэффициенты $C$ будут

$$
\begin{aligned}
& C_{1,2}= \pm \frac{\dot{x}_{0}-\frac{\omega p}{2}}{a_{1 x}-a_{2 x}} \\
& C_{3,4}= \pm \frac{\dot{y}_{0}-\omega\left(x_{0}-x_{\mathrm{c}}\right)}{a_{1 y}-a_{2 y}}
\end{aligned}
$$

Следовательно,

$$
\begin{gathered}
x=x_{0}+\frac{\omega p t}{2}+\frac{\dot{x}_{0}-\frac{\omega p}{2}}{a_{1 x}-a_{2 x}}\left(e^{\alpha_{11} t}-e^{\alpha_{2} x^{t}}\right) \\
y=y_{0}+\omega\left(x_{0}-x_{\mathrm{c}}\right)+\frac{\omega^{2} p t^{2}}{4}+\frac{\dot{y}_{0}-\omega\left(x_{0}-x_{\mathrm{c}}\right)}{a_{1 y}-a_{2 y}}\left(e^{\alpha_{1} t}-e^{\alpha_{2} y^{t}}\right) .
\end{gathered}
$$

Отсюда видно, что при СС второго порядка полное устранение динамической ошибки возможно лишь при $\dot{x}_{0}=\frac{\omega p}{2}$ и $\dot{y}_{0}=\omega\left(x_{0}-x_{\mathrm{c}}\right)$.

Возьмем $x_{0}=y_{0}=x_{\mathrm{c}}=y_{\mathrm{c}}=0$. Оценим ошибку, если начальные скорости $\dot{x}_{0}=\dot{y}_{0}=0$, т. е. системы подач до начала отработки данной параболы были остановлены.

Из (23) получим (для идентичных СС)

$$
y=\frac{x^{2}}{p}+\frac{\omega^{2} p}{4}\left(2 t-\frac{e^{\alpha_{1} t}-e^{\alpha_{2} t}}{a_{1}-a_{2}}\right) \frac{e^{\alpha_{1} t}-e^{\alpha_{2} t}}{a_{1}-a_{2}} .
$$


Чтобы оценить возможный максимум ошибки, надо найти максимум функции

$$
f(t)=\left(2 t-\frac{e^{\alpha_{1} t}-e^{\alpha_{2} t}}{a_{1}-\alpha_{2}}\right) \frac{e^{\alpha_{1} t}-e^{\alpha_{2} t}}{\alpha_{1}-\alpha_{2}} .
$$

Такой способ коррекции программы, где после коррекции $x=x_{\mathrm{I}}$ и $y=y_{1}$, не единственный. Возможен и такой способ, при котором $x \neq x_{1}$ и $y \neq y_{1}$, но изображающая точка находится на заданной параболе. Если, например, ввести в ПУ описываемые уравнением (22) ( $x_{\mathrm{c}}=$ $\left.=y_{\mathrm{c}}=0\right)$ начальные условия

$$
\begin{aligned}
& X_{0}=x_{0} \\
& y_{0}=y_{0}+\frac{\omega^{2} p T_{1 x}}{4}+\frac{\omega^{2} p T_{1 y}}{2}+\omega x_{0} T_{1 y},
\end{aligned}
$$

тогда изображающая точка движется по заданной параболе. Общее решение будет иным, чем (23).

\section{Воспроизведение окружностей}

На выходе ПУ имеем

$$
\begin{aligned}
& x=x_{\mathrm{c}}+\left(y_{0}-y_{\mathrm{c}}\right) \sin \omega t+\left(x_{0}-x_{\mathrm{c}}\right) \cos \omega t . \\
& y=y_{\mathrm{c}}-\left(x_{0}-x_{\mathrm{c}}\right) \sin \omega t+\left(y_{0}-y_{\mathrm{c}}\right) \cos \omega t .
\end{aligned}
$$

Можем писать

$$
\begin{aligned}
& x-x_{\mathrm{c}}=R_{0} \sin \left(\omega t+\varphi_{0}\right) \\
& y-y_{\mathrm{c}}=R_{0} \cos \left(\omega t+\varphi_{0}\right),
\end{aligned}
$$

где $R_{3}=\sqrt{\left(x_{0}-x_{\mathrm{c}}\right)^{2}+} \overline{\left(y_{0}-y_{\mathrm{c}}\right)^{2}}$ и $\varphi_{0}=\operatorname{arctg} \frac{x_{0}-x_{\mathrm{c}}}{y_{0}-y_{\mathrm{c}}}$.

На выходе СС имеем после окончания переходного процесса:

$$
\begin{aligned}
& x_{1}-x_{\mathrm{c}}=R_{0}\left|\Phi_{1}(j \omega)\right| \sin \left(\omega t+\varphi_{0}+\varphi_{1}\right) \\
& y_{1}-y_{\mathrm{c}}=R_{0}\left|\Phi_{2}(j \omega)\right|^{\prime} \cos \left(\omega t+\varphi_{0}+\varphi_{2}\right) .
\end{aligned}
$$

Здесь $|\Phi(j \omega)|$ - модуль комплексного коэффициента передачи СС; $\varphi_{1}=\arg \left[\Phi_{1}(j \omega)\right] ; \varphi_{2}=\arg \left[\Phi_{2}(j \omega)\right]$.

Если СС идентичные $\left[\Phi_{1}(j \omega)=\Phi_{2}(j \omega)\right]$, тогда уравнения (27) являются параметрическими уравнениями окружности с радиусом

$$
R=R_{0}|\Phi(j \omega)| \text {. }
$$

Следовательно, ошибка воспроизведения окружности

$$
\delta=\Delta R=R_{0}[1-|\Phi(j \omega)|] .
$$

Если СС неидентичные, тогда получим на выходе эллипс. Найдем полуоси и угол поворота осей этого эллипса. 
Обозначаем $R_{0}\left|\Phi_{1}(j \omega)\right|=m ; R_{0}\left|\Phi_{2}(j \omega)\right|=n ; \varphi_{1} \neq \varphi_{2}$.

Переносим начало координат в центр кривой, которая теперь представляется параметрическими уравнениями

$$
\begin{aligned}
& x_{1}=m \sin \left(\omega t+\varphi_{0}+\varphi_{1}\right) \\
& y_{1}=n \cos \left(\omega t+\varphi_{0}+\varphi_{2}\right) .
\end{aligned}
$$

Найдем из этой системы $\sin \omega t$ и $\cos \omega t$. Получим

$$
\begin{aligned}
& \sin \omega t=\frac{\frac{x_{1}}{m} \cos \varphi_{2}-\frac{y_{1}}{n} \sin \varphi_{1}}{\cos \left(\varphi_{2}-\varphi_{1}\right)} \\
& \cos \omega t=\frac{\frac{x_{1}}{m} \sin \varphi_{2}+\frac{y_{1}}{n} \cos \varphi_{1}}{\cos \left(\varphi_{2}-\varphi_{1}\right)} .
\end{aligned}
$$

Возведем в квадрат и сложим. Получим

$$
\frac{x_{1}^{2}}{m^{2}}+\frac{y_{1}^{2}}{n^{2}}+\frac{2 x_{1} y_{1}}{m n} \sin \left(\varphi_{2}-\varphi_{1}\right)=\cos \left(\varphi_{2}-\varphi_{1}\right) \text {. }
$$

Это уравнение эллипса, оси которого повернуты в отношении координатных осей на угол $\alpha$, определяемый из выражения

$$
\operatorname{ctg} 2 \alpha=\frac{n^{2}-m^{2}}{2 m n \sin \left(\varphi_{2}-\varphi_{1}\right)},
$$

н полуоси которого

$$
\begin{gathered}
a=\sqrt{\frac{\frac{\cos \left(\varphi_{2}-\varphi_{1}\right)}{\frac{m^{2}+n^{2}}{2 m^{2} n^{2}} \mp \sqrt{\frac{\left(m^{2}+n^{2}\right)^{2}}{4 m^{4} n^{4}}-\frac{\cos ^{2}\left(\varphi_{2}-\varphi_{1}\right)}{m^{2} n^{2}}}}}{b=\sqrt{\frac{\cos \left(\varphi_{2}-\varphi_{1}\right)}{\frac{m^{2}+n^{2}}{2 m^{2} n^{2}} \pm \sqrt{\frac{\left(m^{2}+n^{2}\right)^{2}}{4 m^{4} n^{4}}-\frac{\cos ^{2}\left(\varphi_{2}-\varphi_{1}\right)}{m^{2} n^{2}}}}}}}
\end{gathered}
$$

(верхние знаки, если $m>n$; нижние, если $n>m$ ). Эти окружности и эллипсы изображены на фиг. 5 .

Вопросы воспроизведения окружностей рассмотрены еще в работах $\left.{ }^{1,2}\right]$. В статье [1] рассмотрен и вопрос о коррекции программы при СС первого и второго порядка.
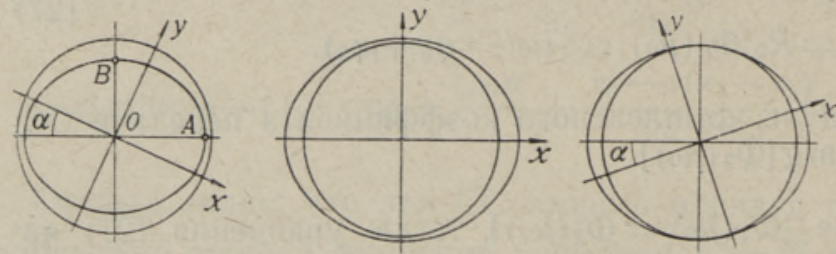

Рассмотрим общие случаи коррекции программы при воспроизведении окружности.

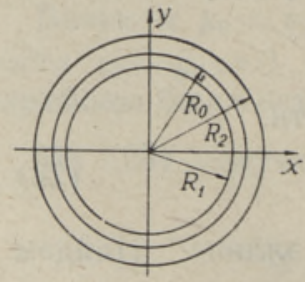

Фиг. 5. Ошибки при воспронзведении окружностей.

$$
\begin{array}{cccc}
m>n & a=O A & m>n & m>n \\
\varphi_{2}>\varphi_{1} & b=O B & \varphi_{2}<\varphi_{1} & \varphi_{2}<\varphi_{1} \\
m=n \text { или } & \left|\Phi_{1}(j \omega)\right|=\left|\Phi_{2}(j \omega)\right|=|\Phi(j \omega)| \\
\text { Если } & |\Phi(j \omega)|>1, \text { то } R=R_{2} \\
& |\Phi(j \omega)|<1 \quad \text {, } R=R_{1}
\end{array}
$$


Желаем иметь на выходе СС окружность (26). В действительности имеем окружность (27).

Чтобы получить на выходе СС (26), на входе СС должно быть $\left(x_{\mathrm{c}}=y_{\mathrm{c}}=0\right)$.

$$
\begin{aligned}
& x=\frac{R_{0}}{\left|\Phi_{1}(j \omega)\right|^{2}} \sin \left(\omega t+\varphi_{0}-\varphi_{1}\right) \\
& y=\frac{R_{0}}{\left|\Phi_{2}(j \omega)\right|^{2}} \cos \left(\omega t+\varphi_{0}-\varphi_{2}\right),
\end{aligned}
$$

где $\Phi(j \omega)=P(\omega)+j Q(\omega) ; \varphi_{1,2}=\arg \left[\Phi_{1,2}(j \omega)\right]=\operatorname{arctg} \frac{Q_{1,2}(\omega)}{P_{1,2}(\omega)}$.

После несложных преобразований получим

$$
\begin{aligned}
& x=\frac{1}{\left|\Phi_{1}(j \omega)\right|^{2}}\left[\left(y_{0} P_{1}(\omega)+x_{0} Q_{1}(\omega)\right) \sin \omega t+\left(x_{0} P_{1}(\omega)-y_{0} Q_{1}(\omega)\right) \cos \omega t\right] \\
& y=\frac{1}{\left|\Phi_{2}(j \omega)\right|^{2}}\left[\left(y_{0} Q_{2}(\omega)-x_{0} P_{2}(\omega)\right) \sin \omega t+\left(y_{0} P_{2}(\omega)+x_{0} Q_{2}(\omega)\right) \cos \omega t\right] .
\end{aligned}
$$

Дифференцируя, получим

$$
\begin{aligned}
& \frac{d x}{d t}=\omega\left(m_{1} \cos \omega t-n_{1} \sin \omega t\right) \\
& \frac{d y}{d t}=\omega\left(q_{1} \cos \omega t-r_{1} \sin \omega t\right) .
\end{aligned}
$$

Выражая из (31) $\sin \omega t$ и $\cos \omega t$ и подставляя их в (32), получим

$$
\begin{aligned}
& \frac{d x}{d t}=\omega\left[\frac{P_{1}(\omega) Q_{2}(\omega)-P_{2}(\omega) Q_{1}(\omega)}{P_{1}(\omega) P_{2}(\omega)+Q_{1}(\omega) Q_{2}(\omega)} x+\frac{\left|\Phi_{3}(j \omega)\right|^{2}}{\left|\Phi_{1}(j \omega)\right|^{2}} \cdot \frac{P_{1}^{2}(\omega)+Q_{1}^{2}(\omega)}{P_{1}(\omega) P_{2}(\omega)+Q_{1}(\omega) Q_{2}(\omega)} y\right] \\
& \frac{d y}{d t}=\omega\left[-\frac{\left|\Phi_{1}(j \omega)\right|^{2}}{\left|\Phi_{2}(j \omega)\right|^{2}} \cdot \frac{P_{2}^{2}(\omega)+Q_{2}(\omega)}{P_{1}(\omega) P_{2}(\omega)+Q_{1}(\omega) Q_{2}(\omega)} x+\frac{P_{1}(\omega) Q_{2}(\omega)-P_{2}(\omega) Q_{1}(\omega)}{P_{1}(\omega) P_{2}(\omega)+Q_{1}(\omega) Q_{2}(\omega)} y\right]
\end{aligned}
$$

или в сокращенном виде

$$
\begin{aligned}
& \frac{d x}{d t}=\omega\left(m_{2} x+n_{2} y\right) \\
& \frac{d y}{d t}=\omega\left(-q_{2} x+r_{2} y\right) .
\end{aligned}
$$

ПУ, описываемое этими дифференциальными уравнениями, усложняется и изображено на фиг. 6.

Фиг. 6. ПУ для коррекции программы ири неидентичных СС.

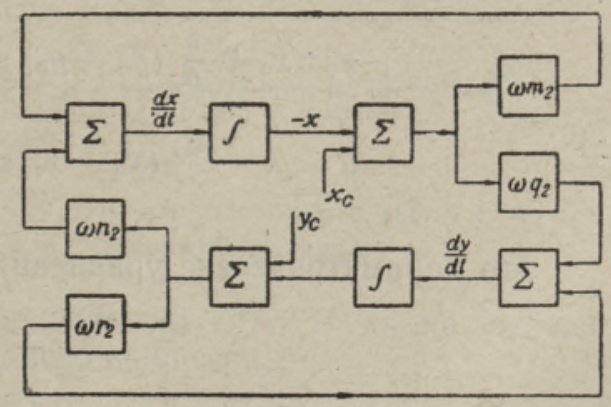


Требуемые начальные условия

$$
\begin{aligned}
X_{0} & =\frac{1}{\left|\Phi_{1}(j \omega)\right|^{2}}\left[-y_{0} Q_{1}(\omega)+x_{0} P_{1}(\omega)\right] \\
Y_{0} & =\frac{1}{\left|\Phi_{2}(j \omega)\right|^{2}}\left[x_{0} Q_{2}(\omega)+y_{0} P_{2}(\omega)\right]
\end{aligned}
$$

Если ПУ идентичные, тогда дифференциальные уравнения ПУ будут -

$$
\begin{aligned}
& \frac{d x}{d t}=\omega y \\
& \frac{d y}{d t}=\omega x .
\end{aligned}
$$

Следовательно, структуры ПУ остаются неизменными (фиг. 1a). Требуемые для коррекции начальные условия описываются теми же уравнениями (34).

В данном случае мы корректировали ошибку как по модулю, так и по аргументу. Но можно корректировать и только по модулю, не корректируя по фазе. В таком случае надо в ПУ, описываемое уравнениями (33), ввести начальные условия

$$
\begin{aligned}
& X_{0}=\frac{1}{\left|\Phi_{1}(j \omega)\right|^{2}}\left(x_{0}-y_{0}\right) \\
& Y_{0}=\frac{1}{\left|\Phi_{2}(j \omega)\right|^{2}}\left(x_{0}+y_{0}\right) .
\end{aligned}
$$

Если $\arg \left[\Phi_{1}(j \omega)\right]=\arg \left[\Phi_{2}(j \omega)\right]$, получим окружность с радиусом $R=R_{0}$. Если аргументы не равны, получим эллипс, оси которого повернуты в отношении координатных осей на $45^{\circ}$ и полуоси

$$
\begin{aligned}
& a=R_{0} \sqrt{\frac{\cos \left(\varphi_{2}-\varphi_{1}\right)}{1-\sin \left(\varphi_{2}-\varphi_{1}\right)}} \\
& b=R_{0} \sqrt{\frac{\cos \left(\varphi_{2}-\varphi_{1}\right)}{1+\sin \left(\varphi_{2}-\varphi_{1}\right)}} .
\end{aligned}
$$

\section{Воспроизведение эллипсов}

На выходе ПУ имеем

$$
\begin{aligned}
& x=x_{\mathrm{c}} \pm \frac{a}{b}\left(y_{0}-y_{\mathrm{c}}\right) \sin \omega t+\left(x_{0}-x_{\mathrm{c}}\right) \cos \omega t \\
& y=y_{\mathrm{c}}-\frac{b}{a}\left(x_{0}-x_{\mathrm{c}}\right) \sin \omega t+\left(y_{0}-y_{\mathrm{c}}\right) \cos \omega t .
\end{aligned}
$$

Это параметрические уравнения эллипса

$$
\frac{\left(x-x_{\mathrm{c}}\right)^{2}}{a^{2}}+\frac{\left(y-y_{\mathrm{c}}\right)^{2}}{b^{2}}=1
$$

(оси эллипса параллельны координатным осям). 
Эти параметрические уравнения можем писать и так:

$$
\begin{aligned}
& x-x_{\mathrm{c}}=a \sin \left(\omega t+\varphi_{0}\right) \\
& y-y_{\mathrm{c}}=b \cos \left(\omega t+\varphi_{0}\right),
\end{aligned}
$$

где $\varphi_{0}=\operatorname{arctg} \frac{b\left(x_{0}-x_{\mathrm{c}}\right)}{a\left(y_{0}-y_{\mathrm{c}}\right)}$.

На выходе СС имеем

$$
\begin{aligned}
& x_{1}-x_{\mathrm{c}}=a\left|\Phi_{1}(j \omega)\right| \sin \left(\omega t+\varphi_{0}+\varphi_{1}\right) \\
& y_{1}-y_{\mathrm{c}}=b\left|\Phi_{2}(j \omega)\right| \cos \left(\omega t+\varphi_{0}+\varphi_{2}\right) .
\end{aligned}
$$

Если следящие системы идентичны, то (по аналогии с окружностью) на выходе СС, получим эллипс с полуосями

$$
\begin{aligned}
& a_{1}=a|\Phi(j \omega)| \\
& b_{1}=b|\Phi(j \omega)| .
\end{aligned}
$$

$$
\text { Ошибки } \begin{aligned}
\Delta a & =a(1-|\Phi(j \omega)|) \\
\Delta b & =b(1-|\Phi(j \omega)|) .
\end{aligned}
$$

Если СС не идентичны, тогда, так же как и в случае окружности, получим эллипс, оси которого повернуты на угол $\alpha . \alpha$ определяется из уравнения (29), а полуоси - из уравнения $(30)$, причем $m=a\left|\Phi_{1}(j \omega)\right|$ и $n=b\left|\Phi_{2}(j \omega)\right|$.

Если, например, $m=n$, то вместо эллипса получим окружность с радиусом $R=a\left|\Phi_{1}(j \omega)\right|=b\left|\Phi_{2}(j \omega)\right|$.

Коррекция программы при воспроизведении эллипсов аналогична с коррекцией программы при окружностях.

\section{Воспроизведение парабол 3 и 4 степени}

Рассмотрим параболы, ось которых совпадает с осью $у$ и вершина или точка перегиба находится в начале координат. Уравнения парабол третьей степени $y=\frac{x^{3}}{p}$ четвертой степени $y=\frac{x^{4}}{p}$.

Соответствующее ПУ изображено на фиг. 7.

При параболе третьей степени имеем на выходе Пу

$$
\begin{aligned}
& x=\omega p t \\
& y=\omega^{3} p^{2} t .
\end{aligned}
$$
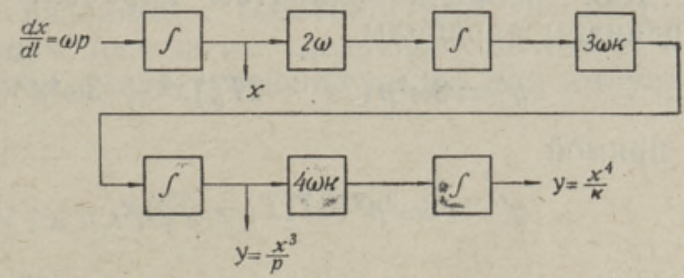

Фиг. 7. ПУ для парабол третьего и четвертого порядка.

Пусть СС снова описывается уравнениями (2). Тогда на выходе СС имеем (после окончания переходного процесса):

$$
\begin{aligned}
& x_{1}=\omega p t-\omega p T_{1} \\
& y_{1}=\omega^{3} p^{2} t^{3}-3 \omega^{3} p^{2}\left(T_{1} t^{2}+2 T_{2}^{2} t-2 T_{1}^{2} t+2 T_{1}^{3}-4 T_{1} T_{2}^{2}\right) .
\end{aligned}
$$


Уравнение кривой на выходе СС

$$
y=\frac{x^{3}}{p}=3 \omega^{2} p\left(T_{1}^{2}-2 T_{2}^{2}\right) x+2 \omega^{3} p^{2}\left(3 T_{1} T_{2}^{2}-T_{1}^{3}\right) .
$$

Это парабола третьей степени. Поведение ее зависит от знака выражения

$$
\Delta=9 \omega^{2}\left(T_{1}^{2}-27_{2}^{2}\right) .
$$

Если $\Delta \geqslant 0$, кривая не имеет экстремумов, при $\Delta<0$ кривая имеет один максимум и один минимум.

Координаты экстремумов

$$
\begin{aligned}
& x_{\text {экс }}=\mp \omega p \sqrt{\mp\left(2 T_{2}^{2}-T_{1}^{2}\right)} \\
& y_{\text {экс }}=2 \omega^{3} p^{2}\left[\left(3 T_{1} T_{2}^{2}-T_{1}^{3}\right) \pm\left(2 T_{2}^{2}-T_{1}^{2}\right) \sqrt{\mp\left(2 T_{2}^{2}-T_{1}^{2}\right)}\right] \approx \delta_{\max } .
\end{aligned}
$$

Координаты точки перегиба

$$
x_{\mathrm{c}}^{\prime}=0 ; \quad y_{\mathrm{c}}^{\prime}=2 \omega^{3} p^{2}\left(3 T_{1} T_{2}^{2}-T_{1}^{3}\right) .
$$

Знаки зависят от знака $\Delta$ и от знака отношения $\beta=\frac{T_{1}^{2}}{T_{2}^{2}}$.

При параболе четвертой степени на выходе ПУ имеем

$$
\begin{aligned}
& x=\omega p t \\
& y=\omega^{4} p^{3} t^{4} .
\end{aligned}
$$

На выходе СС имеем

$$
\begin{aligned}
& x_{1}=\omega p t-\omega p T_{1} \\
& y_{1}=\omega^{4} p^{3} t^{4}-4 \omega^{4} p^{3}\left[T_{1} t^{3}+3\left(T_{1}^{2}-T_{2}^{2}\right) t^{2}-6\left(T_{1}^{2}-2 T_{2}^{2} T_{1}\right) t+6 T_{1}^{4}-\right. \\
& \left.\quad-18 T_{2}^{2} T_{1}^{2}+6 T_{2}^{4}\right) .
\end{aligned}
$$

Уравнение кривой на выходе СС:

$$
\begin{gathered}
y=\frac{x^{4}}{p}+6 \omega^{2} p\left(T_{1}^{2}-2 T_{2}^{2}\right) x^{2}+8 \omega^{3} p^{2}\left(3 T_{2}^{2} T_{1}-T_{1}^{3}\right) x+ \\
+3 \omega^{4} p^{3}\left(3 T_{1}^{4}+8 T_{2}^{4}-12 T_{1}^{2} T_{2}^{2}\right) .
\end{gathered}
$$

Как видно, к ординатам параболы на выходе ПУ прибавляются ординаты параболы

$$
y^{\prime}=6 \omega^{2} p\left(T_{1}^{2}-2 T_{2}^{2}\right) x^{2}+3 \omega^{4} p^{3}\left(3 T_{1}^{4}+8 T_{2}^{4}-12 T_{1}^{2} T_{2}^{2}\right)
$$

и прямой

$$
y^{\prime \prime}=8 \omega^{3} p^{2}\left(3 T_{2}^{2} T_{1}-T_{1}^{3}\right) x .
$$

В зависимости от отношения $\beta_{1}=\frac{T_{1}^{2}}{T_{2}^{2}}$ парабола $y^{\prime}$ и прямая $y^{\prime \prime}$ могут принимать разные положения. При $\beta_{1}<2$ парабола $y^{\prime}$ обращена вершиной вверх; при $\beta_{1}>2$ парабола $y^{\prime}$ обрацена вершиной вниз. При $\beta_{1}<0,85$ вершина параболы $y^{\prime}$ находится выше вершины параболы $y$ на выходе ПУ; при $0,85<\beta_{1}<3,15$ вершина $y^{\prime}$ находится ниже, а при $\beta_{1}>3,15$ выше, чем вершина заданной параболы $y$. Прямая $y^{\prime \prime}$ имеет 
положительный наклон, если $\beta_{1}<3$ и отрицательный наклон, если $\beta_{1}>3$. Если высшие постоянные времени $T_{3} \neq-0$ и $T_{4} \neq 0$, то эти соотношения, конечно, меняются.

Следовательно, в зависимости от $\beta_{1}$ кривая на выходе СС может принимать разные положения в отношении параболы на выходе ПУ.

\section{Заключение}

Рассмотрение динамических ошибок двухкоординатных СС показывает, что эти ошибки существенны при больших скоростях. При воспроизведении прямых линий динамическая ошибка пропорциональна скорости движения по траектории, а при воспроизведении кривых второго порядка - квадрату скорости, при параболе третьего и четвертого порядков соответственно третьей и четвертой степеням скорости.

Увеличение ошибок по отдельным координатам не всегда увеличивает динамическую ошибку воспроизведения, так же как и уменьшение ошибок по отдельным координатам не всегда их уменьшает. Если. например, при воспроизведении параболы $y=\frac{x^{2}}{p}$ ликвидировать динамическую ошибку по координате $x$ и уменьшить ее по координате $y$ путем введения в СС кроме управляющего сигнала еще его первой производной, то ошибка воспроизведения после окончания переходного процесса растет по сравнению с некорректированной системой.

Для коррекции динамических ошибок имеется два пути:

1) уменьшить динамические ошибки по отдельным координатам в такой мере, чтобы ошибка воспроизведения была меньше заданной;

2) обеспечить такое соотношение между $\Delta x$ и $\Delta y$, чтобы изображающая точка находилась все время на заданной кривой, но при этом $x \neq x_{1}$ и $y \neq y_{1}$.

Уменьшение динамических ошибок путем коррекции программы возможно, если параметры СС известны. Но если СС неидентичны, ПУ усложняется, что нежелательно. Поэтому при таком способе коррекции следует рекомендовать следящие системы делать идентичными. Ошибку переходного процесса при коррекции программы можно ликвидировать только при помощи подходящего выбора начальных скоростей, что не всегда возможно.

Помимо коррекции программы надо искать пути улучшения динамических свойств самих СС, связанные с особенностями двухкоординатных СС с ПУ. Этому и будут посвящены следующие статьи, в которых полученные здесь решения будут уточнены также для импульсных систем.

\section{ЛИТЕРАТ У Р А}

1. А. А. Ворояов, О применении цифровых аналогов для программирования кривых второго порядка в двухкоординатных системах автоматического управления. Сборник работ по вопросам электромеханикн, вып. III, Изд. АН СССР, 1960.

2. Б. И. Анд рейчиков, Динамическая точность станков с ПУ. Доклад на конференции молодых специалистов КАТ АН СССР, 1960.

Ннститут кибернетики

Академии наук Эстонской ССР
Поступила в редакцию

9. XII 1960 


\section{KAHE KOORDINAADIGA PROGRAMMJUHTIMISEGA JÄLGIVSUSTEEMIDE DUNAAMILISTEST VIGADEST}

\section{K. Leppik \\ Resümee}

Artiklis vaadeldakse dünaamilisi vigu, mis esinevad sirgete, ringide, ellipsite ning ruut-, kuup- ja neljanda astme paraboolide töötlemisel kahe koordinaadiga programmjuhtimisega jälgivsüsteemi abil. Viimast saab kirjeldada teist järku diferentsiaalvôrranditega.

Antakse valemid vigade arvutamiseks ja näidatakse moodus nende vigade kõrvaldamiseks programmi korrigeerimise teel. Esitatakse algtingimuste parandused, mida tuleb arvestada programmi koostamisel.
Eesti NSV Teaduste Akadeemia
Saabus toimetusse
Küberneetika Instituut
9. XII 1960

\section{OBER DYNAMISCHE FEHLER IN FOLGEREGELUNGSSYSTEMEN MIT PROGRAMMSTEUERUNG (MIT ZWEI KOORDINATEN)}

\section{K. Leppik}

\section{Zusammenjassung}

Der Artikel behandelt dynamische Fehler, welche bei der Verarbeitung der Geraden, Kreise, Ellipsen und Parabolen der zweiten, dritten und vierten Potenz in Folgeregelungssystemen mit Programmsteuerung (mit zwei Koordinaten) entstehen können. Die letzteren können durch Differentialgleichungen der zweiten Stufe beschrieben werden.

Der Verfasser gibt Formeln zur Berechnung der Fehler und zeigt Wege zu deren Berichtigung durch Korrigieren des Programms. Es werden Korrekturen der Ausgangsbedingungen gebracht, die bei der Zusammenstellung des Programms zu berücksichtigen wären. 